\title{
Effects of oxymetazoline nasal spray on the nasal cycle assessed by long-term rhinoflowmetry*
}

\author{
Thomas Braun, Maria Rich, Alexander Berghaus, Matthias F. Kramer \\ Rhinology 50: 370-375, 2012 \\ DOI:10.4193/Rhino12.032 \\ Department of Otorhinolaryngology, Head and Neck Surgery, Ludwig Maximilian University, Munich, Germany \\ *Received for publication: \\ February 16,2012 \\ Accepted: May 30, 2012
}

\section{Summary}

Background: Long-term rhinoflowmetry assesses bilateral nasal flow over 24 hours. In the present study, we evaluated the effects of a standard dose of oxymetazoline topical nasal spray, a widely used over-the-counter drug, on the nasal cycle, since the exact long-term effects, such as the duration of the decongestive effect, are not yet reported.

Methodology/Principal: Thirty healthy volunteers received a portable long-term rhinoflowmetry device and applied $22.5 \mu \mathrm{g}$ oxymetazoline in each nostril.

Results: In $90 \%$ of the probands, effects of the nasal spray application could be seen as changes in nasal flow. A decongestive effect could be seen after 18 minutes on average. We found a mean duration of the maximal decongestive effect of four hours. However, it took more than six hours on average until the nasal cycle resumed its normal condition. We did not find significant differences of the effect between probands with a 'classic,'in concert' or impaired nasal cycle. In contrast to a substantial interindividual variability, repeated measurements showed that intraindividual variability of the effect of decongestive nasal spray seems to be rather small.

Conclusion: Long-term rhinoflowmetry, yielding reliable results, is a valuable tool in the assessment of the effects of nasal drugs on the nasal cycle.

Key words: long-term rhinoflowmetry, nasal cycle, decongestive nasal spray, oxymetazoline

\section{Introduction}

Objective apparative methods to assess the respiratory function of the nose can be used in preoperative diagnostics before functional or aesthetic rhinosurgery and as a postoperative quality control ${ }^{(1)}$. Flow resistance and dynamics can be measured by rhinomanometry and rhinoresistometry, while acoustic rhinometry describes the geometry of the nasal flow channel ${ }^{(1,2)}$. However, with all these methods, only a momentary record of nasal function is achieved. Therefore, long-term rhinoflowmetry was developed to assess nasal function also under everyday life conditions and during sleep: Using tubes inserted bilaterally in the nasal vestibule, pressure fluctuations are measured over 24 hours and stored in a portable battery-powered device, which allows visualization of bilateral changes in nasal flow ${ }^{(3)}$. Currently, long-term rhinoflowmetry can be considered the most valuable method to investigate long-term changes in the nasal cycle and pathological states of congestion under everyday life conditions ${ }^{(1)}$.

We thought that long-term rhinoflowmetry could also be a valuable tool in the assessment of effects of topical drugs on the nasal cycle and initiated the present study. As a test substance, 
we chose oxymetazoline, which, as topical nasal spray, is a widely used over-the-counter drug e.g. for the therapy of common cold ${ }^{(4,5)}$. Oxymetazoline is an agonist at sympathetic a1 receptors, therefore causing vasoconstriction and decongestion (6). Additionally, we were interested to know if the predominant type of nasal cycle present in the proband had any correlation with the measured effects.

\section{Materials and methods}

\section{Subjects}

The study was approved by the ethics commission of the medical faculty, Ludwig Maximilian University, Munich.

In 30 healthy volunteers ( 14 women, 16 men, mean age 29 years, range 19 - 58 years) without subjectively impaired nasal breathing, rhinomanometry, rhinoresistometry and acoustic rhinometry were performed before and after nasal decongestion for both sides. All measurements were conducted with the Rhino-Sys diagnostic system (Happersberger otopront $\mathrm{GmbH}$, Hohenstein, Germany) according to the recommendations of the 'International Committee on the Objective Assessment of the Upper Airways ${ }^{(2)}$.' In all subjects, relevant pathologies such as more than a slight septal deviations, hyperplastic turbinates or polyposis had been ruled out by anterior rhinoscopy and nasal endoscopy. There was no history of nasal spray abuse or regular smoking, and patients did not have any nasal symptoms.

\section{Rhinoflowmetry}

After these preparations, the long-term rhinoflowmetry device (Happersberger otopront $\mathrm{GmbH}$, Hohenstein, Germany, Figure 1) was calibrated according to the instructions of the manufacturer and handed out to the proband together with oxymetazo- line nasal spray (Nasivin ${ }^{\oplus}$, Merck, Darmstadt, Germany, with one dose containing $22.5 \mu$ g oxymetazoline hydrochloride). Patients were instructed to start the device at a convenient time at home the next day and apply a single dose of nasal spray in each nostril 3-5 hours later. Upon return of the device, the obtained data were retrieved (example shown in Figure 2) and entered into a statistical spreadsheet for further analysis.

\section{Statistical analysis}

Descriptive statistics and comparison between groups by t-test for independent samples or Mann-Whitney test after testing for normal distribution with Shapiro-Wilk test were computed by SPSS Statistics 17.0 (SPPS Inc., Chicago, IL, USA). For the assessment of repeated mesasurement, t-test for dependent sample was used.

In five patients, a second measurement was performed to assess intraindividual variability of the determined effects.

\section{Results}

Rhinoresistometry and acoustic rhinometry

Descriptive statistics of the objective variables of nasal patency for the right and left side before and after decongestion are demonstrated in Table 1. The collective had a mean hydraulic diameter of 4.0 - $4.2 \mathrm{~mm}$ before and 5.5 - $5.6 \mathrm{~mm}$ after decongestion. The collective had mean minimal cross-sectional areas of $0.8 \mathrm{~cm}^{2}$ (MCA1) and $1.9-2.0 \mathrm{~cm}^{2}$ (MCA2) before decongestion. After decongestion, $0.9-1.0 \mathrm{~cm}^{2}$ (MCA1) and $3.0 \mathrm{~cm}^{2}$ (MCA2) were calculated.

\section{Long-term rhinoflowmetry}

Of the 30 probands, 15 (50\%) had a predominant 'classic' nasal

Table 1. Objective variables of nasal patency for the right and left side determined in all subjects before and after decongestion.

\begin{tabular}{|c|c|c|c|c|c|c|c|}
\hline Variable & Side & Decongestion & Mean & Median & Minimum & Maximum & SD \\
\hline \multirow{5}{*}{ HD [mm] } & \multirow[t]{2}{*}{ Right } & Before & 4.2 & 4.1 & 2.9 & 5.6 & 0.8 \\
\hline & & After & 5.6 & 5.5 & 3.6 & 7.0 & 0.9 \\
\hline & \multirow[t]{2}{*}{ Left } & Before & 4.0 & 3.9 & 2.7 & 5.5 & 0.8 \\
\hline & & After & 5.5 & 5.7 & 3.7 & 8.2 & 1.0 \\
\hline & \multirow[t]{2}{*}{ Right } & Before & 0.8 & 0.8 & 0.4 & 1.5 & 0.3 \\
\hline \multirow{3}{*}{$\mathrm{MCA} 1\left[\mathrm{~cm}^{2}\right]$} & & After & 1.0 & 1.0 & 0.5 & 2.1 & 0.4 \\
\hline & \multirow[t]{2}{*}{ Left } & Before & 0.8 & 0.8 & 0.4 & 1.5 & 0.3 \\
\hline & & After & 0.9 & 0.9 & 0.5 & 1.5 & 0.3 \\
\hline \multirow{4}{*}{$\operatorname{MCA} 2\left[\mathrm{~cm}^{2}\right]$} & \multirow[t]{2}{*}{ Right } & Before & 1.9 & 1.9 & 1.0 & 3.6 & 0.6 \\
\hline & & After & 3.0 & 2.9 & 1.9 & 4.6 & 0.7 \\
\hline & \multirow[t]{2}{*}{ Left } & Before & 2.0 & 2.0 & 0.7 & 3.6 & 0.7 \\
\hline & & After & 3.0 & 3.1 & 1.5 & 4.3 & 0.7 \\
\hline
\end{tabular}

$(\mathrm{HD}=$ hydraulic diameter; $\mathrm{MCA}=$ minimal cross-sectional area; $\mathrm{SD}=$ standard deviation $)$. 
Table 2. Long-term rhinoflowmetry.

\begin{tabular}{|c|c|c|c|c|c|c|c|c|c|}
\hline \multirow{4}{*}{$\begin{array}{l}\text { Latency of } \\
\text { decongestive } \\
\text { effect [min] }\end{array}$} & All probands & 0 & 59 & 18 & 18 & 16 & & & \\
\hline & 'Classic' type & 0 & 59 & 18 & 21 & 16 & \multirow{3}{*}{0.296} & \multirow{3}{*}{0.312} & \multirow{3}{*}{0.628} \\
\hline & 'In concert' type & 0 & 27 & 18 & 14 & 8 & & & \\
\hline & Impaired type & 0 & 54 & 5 & 15 & 20 & & & \\
\hline \multirow{4}{*}{$\begin{array}{l}\text { Duration } \\
\text { of maximal } \\
\text { decongestive } \\
\text { effect [min] }\end{array}$} & All probands & 9 & 882 & 216 & 242 & 229 & & & \\
\hline & 'Classic' type & 9 & 882 & 248 & 293 & 252 & \multirow{3}{*}{0.360} & \multirow{3}{*}{0.239} & \multirow{3}{*}{0.945} \\
\hline & 'In concert' type & 9 & 531 & 216 & 198 & 175 & & & \\
\hline & Impaired type & 14 & 576 & 68 & 174 & 196 & & & \\
\hline \multirow{4}{*}{$\begin{array}{l}\text { Latency until } \\
\text { resumed nasal } \\
\text { cycle [min] }\end{array}$} & All probands & 9 & 1112 & 324 & 372 & 302 & & & \\
\hline & 'Classic' type & 9 & 1112 & 367 & 463 & 332 & \multirow{3}{*}{0.134} & \multirow{3}{*}{0.369} & \multirow{3}{*}{0.576} \\
\hline & 'In concert' type & 9 & 531 & 225 & 237 & 203 & & & \\
\hline & Impaired type & 54 & 711 & 243 & 316 & 248 & & & \\
\hline
\end{tabular}

Latency of the decongestive effect, duration of the maximal decongestive effect, and latency until the nasal cycle was resumed for all probands with an effect of application of one dose of $22.5 \mu \mathrm{g}$ oxymetazoline nasal spray per nostril $(n=27)$ as well as for the subgroups with a dominating 'classic' $(n=14)$, 'in concert' $(n=7)$ or impaired nasal cycle $(n=6)$. SD = standard deviation; $p=p$ value, t-test for independent samples (normally distributed values) or Mann-Whitney test (not normally distributed values, as verified by Shapiro-Wilk test).

cycle with both sides alternating between a congestive and decongestive state. In 8 probands (27\%), an 'in concert' nasal cycle was found, with both sides concomitantly alternating between a congestive and decongestive state. Interestingly, for shorter periods of time, mostly during the day, in 6 of the probands with a dominating 'classic' nasal cycle, also 'in concert' phases were found. Accordingly, in 3 of the probands with a predominant 'in concert' nasal cycle, shorter 'classical' phases were found mostly during sleep. An impaired nasal cycle, where no systematic alterations between the congestive and decongestive state were found, was present in the remaining 7 probands (23\%).

In 27 probands (90\%) an effect of the nasal spray application (one spray per nostril) could be seen as changes in nasal flow, while in 3 probands (10\%) no effect was present. In the probands with an effect present, we analyzed the latency of the decongestive effect, the duration of the maximal decongestive effect, and the latency until the nasal cycle was resumed. Table 2 and Figure 3 show the respective times for all probands as well as for the subgroups with a predominant 'classic,'in concert' or an impaired nasal cycle. In the whole collective, a decongestive effect could be seen after 18 minutes on an average. We found a mean duration of the maximal decongestive effect of 242 minutes, and it took 372 minutes on an average until the nasal cycle resumed its normal condition. Statistical comparison did not show any significant differences of these durations between the subgroups (Table 2).

In 5 probands with an effect of the nasal spray application present, a second measurement over 24 hours was performed to assess intraindividual variability. Statistical comparison did not show significant differences concerning latency of the decongestive effect, the duration of the maximal decongestive effect (Figure $4 a, b$ ). Concerning the latency until the nasal cycle was resumed, there was a difference of this variable by trend (Figure 4c), but without statistical significance.

\section{Discussion}

The nasal cycle, i.e. the bilateral cyclic fluctuation of the congestive state of the nasal mucosa, was first described by Richard Kayser in $1895^{(7,8)}$. In $13-80 \%$ of adults, a 'classic'type is found with identical bilateral periods of the cycle, but 180 degrees out of phase ${ }^{(9)}$, while an 'in concert' type with both nasal sides in phase is thought to be the "working phase" for the whole nose ${ }^{(1)}$. However, the exact function of the nasal cycle is still not fully understood ${ }^{(10)}$. Eccles proposes that it plays a major part in respiratory defence ${ }^{(11)}$. The duration of the nasal cycle varies from 30 minutes to six hours ${ }^{(9)}$. In our collective, a 'classic' nasal cycle was present in $50 \%$. Interestingly, we found an 'in concert' type in some of these patients during the day, which supports the concept of the 'in concert' type as the 'working phase' of the nose. Vice versa, some patients with a predominant 'in concert' type during the day switched to a 'classic' type during sleep. The underlying physiological mechanisms are not clear.

The objective data obtained by acoustic rhinometry (Table 1) is in accordance with the clinical examination ruling out obvious 

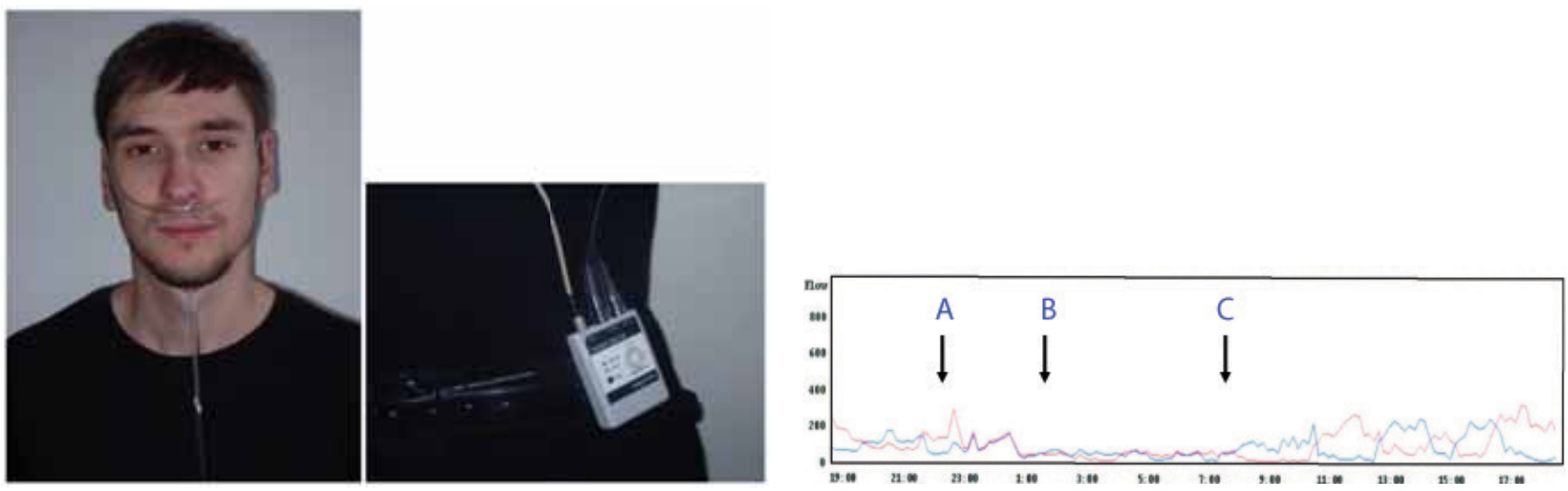

Figure 1. Setup of the portable long-term rhinoflowmetry device.

Figure 2. Example of acquired data over 24 hours (bilateral nasal flow). A: Application of the nasal spray ( $22.5 \mu \mathrm{g}$ oxymetazoline per nostril). B: End of the maximal decongestive effect. C: Normal nasal cycle resumed.

obstructive nasal pathologies. Acoustic rhinometry analyzes the reflection of acoustical signals to objectify the geometry of the inner nose. MCA1 and MCA2 are the minimal cross-sectional areas at the typical two narrowest locations, the nasal isthmus and the head of the inferior turbinate and cavernous body of the nasal septum, respectively. Mlynski reports that after decongestion, a normal MCA1 should not be below $0.5 \mathrm{~cm}^{2}$, and a nor-

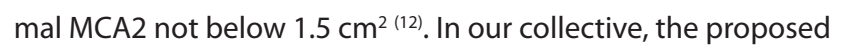
normal values were reached already in the not-decongested state. In rhinoresistometry, which is basically the calculation of additional variables from rhinomanometry by laws of fluid dynamics, the variable 'hydraulic diameter,'HD, is used to describe nasal patency ${ }^{(1)}$. HD is the diameter of an imaginary round pipe with the same flow resistance as the nose of the measured subject. While no internationally accepted normal values are yet established, Mlynski recommends to consider a HD $<5.5 \mathrm{~mm}$ as too narrow and $>6.5 \mathrm{~mm}$ as too wide, while normal nasal patency is thought to lie between these cut-off values ${ }^{(12)}$. In our collective, in contrast, HD did not exceed $5.5 \mathrm{~mm}$ before and after decongestion, maybe suggesting that Mlynski's proposed normal value for HD is somewhat too high. Since clinical examination and acoustic rhinomanometry yielded normal results, and no internationally accepted normal values are known for $\mathrm{HD}$, we think that the collective can be considered lacking relevant nasal pathologies concerning the study. However, since the distribution of the volunteers' age in this study was quite skew with a mean age of 29, may maybe the results cannot be transferred to patients of any age, especially older people.

In $10 \%$ of the probands, no effect of the applied nasal spray could be visualized by long-term rhinoflowmetry. The reason could be a too small dose for the individual, most probable due to faulty application of the spray by the proband. With correct application, $100 \%$ of a nasal pump spray is also deposited in the nose ${ }^{(13)}$. Extreme septal deviations or nasal valve stenosis were not present in the present collective.

A decongestive effect could be seen after 18 minutes on average. This is congruent with Bickford's report of changes in nasal resistance and the sum of minimal cross-sectional areas 15 minutes after the application of oxymetazoline nasal spray in 20 volunteers ${ }^{(14)}$. We found a mean duration of the maximal decongestive effect of 242 minutes, i.e. four hours. Since Bickford found an effect still present after two hours, but quit his obtaining data afterwards, this is, to the best knowledge of the authors, the first data based on exact measurements about the duration of the decongestive effect of oxymetazoline nasal spray. An end of the maximal decongestive effect after roughly four hours is also in good correlation to the subjective perception after the appliance of decongestive nasal spray (own clinical observations). However, it took more than six hours on average until the nasal cycle resumed its normal condition. In a single case, we found that the application of only one dose per nostril impaired the nasal cycle for more than 18 hours (Table 3 ). The extent of the standard deviation also shows that there is a substantial interindividual variability of the effect of decongestive nasal spray. This might be the explanation why there are different reports in the literature about the latency of side effects of recurrent use of decongestive nasal spray, e.g. the development of rhinitis medicamentosa ${ }^{(4,15)}$. However, we did not found significant differences of the effect between probands with a 'classic,'in concert' or impaired nasal cycle (Table 2, Figure 3). This is most probable be explained by the fact that oxymetazoline has a direct pharmacological effect on the adrenoreceptors of the blood vessels ${ }^{(6)}$, and its effect should not be influenced by nerval control.

Bickford et al., evaluated the effects of oxymetazoline nasal 
a

\section{Latency of the decongestive effect}

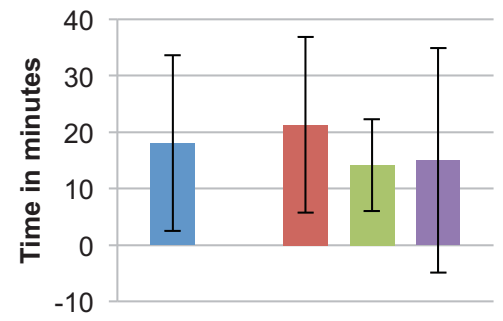

All probands

Classic type

In concert type

- Impaired type

$-10$

b

Maximal decongestive effect

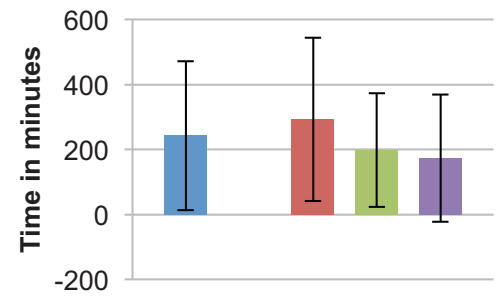

- All probands

Classic type

- In concert type

- Impaired type

C

\section{Latency until nasal cycle resumed}

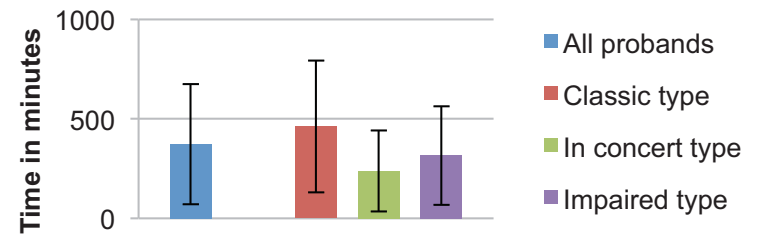

Figure 3. Long-term rhinoflowmetry: latency of the decongestive effect (a), duration of the maximal decongestive effect (b), and latency until the nasal cycle was resumed (c) for all probands with an effect of application of one dose of $22.5 \mu \mathrm{g}$ oxymetazoline nasal spray per nostril (n $=27$ ) as well as for the subgroups with a dominating 'classic' $(n=14)$, 'in concert $(n=7)$ or impaired type $(n=6)$ of the nasal cycle. Small bars: standard deviation.

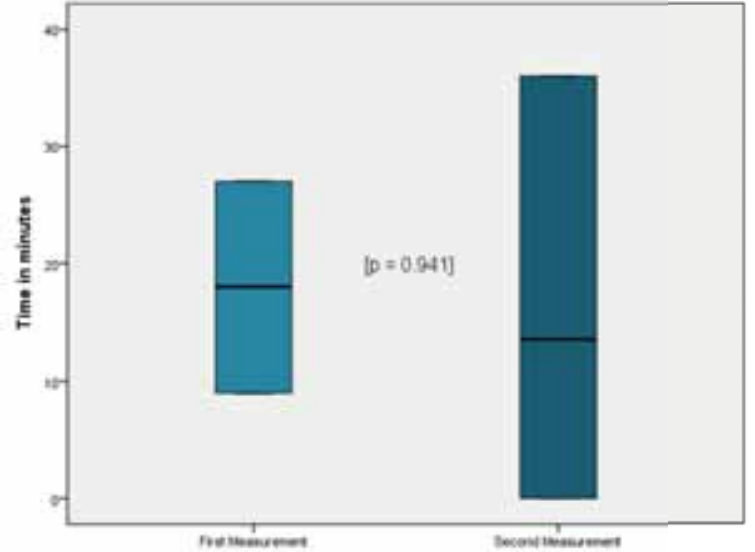

b

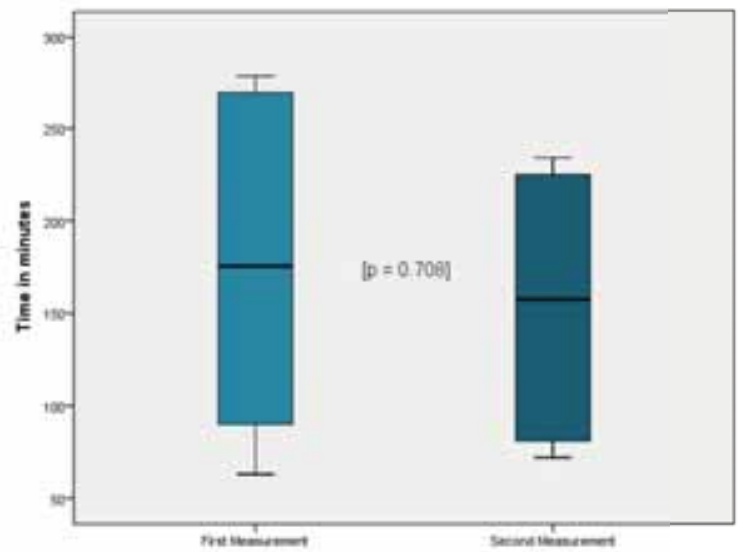

C

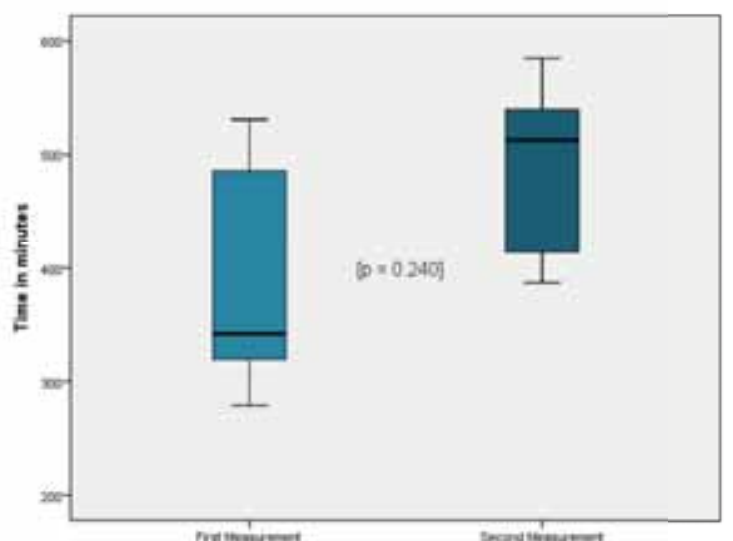

Figure 4. Long-term rhinoflowmetry: intraindividual variability of the latency of the decongestive effect (a), the duration of the maximal decongestive effect (b), and the latency until the nasal cycle was resumed (c) for five probands with an effect of application of one dose of $22.5 \mu \mathrm{g}$ oxymetazoline nasal spray per nostril. Box-and-whiskers plot. $p=p$ value, t-test for independent samples. spray on nasal patency in healthy subjects using rhinomanometry and acoustic rhinometry. After bilateral application of 0.9 mg oxymetazoline, nasal resistance decreased, while the sum of the minimal cross-sectional areas increased ${ }^{(14)}$. However, since measurements were only obtained up to 120 minutes after application of the spray, long-term effects, such as duration of the 
decongestive effect, remain unknown from this study.

Eccles et al., compared xylometazoline nasal spray with placebo and found significant effects on nasal conductance for at least 10 hours after application in patients with a common cold, not regarding healthy subjects ${ }^{(16)}$. Soubeyrand extensively studied the effect of different vasoconstrictive drugs on the nasal cycle by rhinomanometry. In 25 probands with healthy mucosa and rhinosinusitis, he found duration effects for different imidazoles (comparable to oxymetazoline used in this study) of $5-7$ hours in healthy subjects and $3.5-6$ hours under pathologic conditions. This is comparable with the result of this study that oxymetazoline has effects on the nasal cycle for more than six hours in healthy subjects; as suggested by the results of Soubeyrand, effects of drugs applied to the nasal mucosa might be shorter in inflammatory conditions due to increased blood flow ${ }^{(17)}$.

While Soubeyrand had to perform multiple rhinomanometric measurements for assessing the nasal cycle over several ours in his study from $1964{ }^{(17)}$, long-term rhinoflowmetry as described in this study seems to be a more comfortable alternative to measure the effects of drugs on the nasal cycle under everyday life conditions. The results of the repeated measurements show that in contrast to interindividual variability, intraindividual variability of the effect of decongestive nasal spray seems to be rather small. Additionally, this also means that long-term rhinoflowmetry yielded reliable results with no statistically significant changes of the variables measured. We therefore think that long-term rhinoflowmetry is a valuable tool in the assessment of the effects of nasal drugs on the nasal cycle.

\section{Acknowledgements}

This study received no funding. We thank Happersberger otopront $\mathrm{GmbH}$ for providing the Rhino-Sys system.

\section{Authorship contribution}

T.B.: study design, manuscript preparation; M.R.: measurements, statistical analysis; A.B.: manuscript proofreading; M.F.K.: working group leader, manuscript proofreading.

\section{Conflict of interest}

No conflict of interests.

\section{References}

1. Mlynski G, Beule A. Diagnostic methods of nasal respiratory function. HNO. 2008; 56: 81-99.

2. Clement PA, Gordts F. Consensus report on acoustic rhinometry and rhinomanometry. Rhinology. 2005; 43: 169-179.

3. Grützenmacher S, Lang C, Mlynski R, Mlynski B, Mlynski G. Long-term rhinoflowmetry: a new method for functional rhinologic diagnostics. Am J Rhinol. 2005; 19: 53-57.

4. Watanabe H, Foo TH, Djazaeri B, Duncombe P, Mackay IS, Durham SR. Oxymetazoline nasal spray three times daily for four weeks in normal subjects is not associated with rebound congestion or tachyphylaxis. Rhinology. 2003; 41: 167-174.

5. Akerlund $A$, Klint $T$, Olen L, Rundcrantz $H$. Nasal decongestant effect of oxymetazoline in the common cold: an objective doseresponse study in 106 patients. J Laryngol Otol. 1989; 103: 743-746.

6. Bende M, Loth S. Vascular effects of topical oxymetazoline on human nasal mucosa. J Laryngol Otol. 1986; 100: 285-288.

7. Kayser R. Die exacte Messung der Luftdurchgängigkeit der Nase. Arch Laryngol. 1895; 101-121.

8. Gungor A, Moinuddin R, Nelson RH, Corey JP. Detection of the nasal cycle with acoustic rhinometry: techniques and applications. Otolaryngol Head Neck Surg. 1999; 120: 238-247.
9. Gilbert AN, Rosenwasser AM. Biological rhythmicity of nasal airway patency: a re-examination of the 'nasal cycle'. Acta Otolaryngol. 1987; 104: 180-186.

10. Lund VJ. Nasal physiology: neurochemical receptors, nasal cycle, and ciliary action. Allergy Asthma Proc. 1996; 17: 179-184.

11. Eccles RB. The nasal cycle in respiratory defence. Acta Otorhinolaryngol Belg. 2000; 54: 281-286

12. Mlynski G. Personal communication. 2010

13. Moller W, Saba GK, Haussinger K, Becker S, Keller M, Schuschnig U. Nasally inhaled pulsating aerosols: lung, sinus and nose deposition. Rhinology. 2011; 49: 286-291.

14. Bickford L, Shakib S, Taverner D. The nasal airways response in normal subjects to oxymetazoline spray: randomized doubleblind placebo-controlled trial. Br J Clin Pharmacol. 1999; 48: 53-56.

15. Graf P. Oxymetazoline nasal spray. Laryngoscope. 1998; 108: 1255.

16. Eccles R, Eriksson M, Garreffa S, Chen SC. The nasal decongestant effect of xylometazoline in the common cold. Am J Rhinol. 2008; 22: 491-496

17. Soubeyrand L. Action des médicaments vaso-moteurs sur le cycle nasal et la fonction ciliaire. Rev Laryngol Otol Rhinol (Bord). 1964; 85: 49-113.
Dr. med. Thomas Braun

Klinik und Poliklinik für Hals-Nasen-

Ohrenheilkunde

der Ludwig-Maximilians-Universität

München

Marchioninistraße 15

D-81377 München

Germany

Tel: $+49-8970950$

Fax +49-7095 6869

E-mail: thomas.braun@med.unimuenchen.de 